\section{Effectiveness of a compressive device in controlling hemorrhage following radial artery catheterization}

\author{
Kiho Yun ${ }^{1}$, Woochan Jeon ${ }^{2}$, Bora Kang' ${ }^{1}$, Giwoon Kim ${ }^{1}$ \\ 'Department of Emergency Medicine, Ajou University School of Medicine, Suwon, Korea \\ ${ }^{2}$ Department of Emergency Medicine, Inje University College of Medicine, Ilsan Paik Hospital, Goyang, Korea
}

Objective The purpose of this study was to evaluate the effectiveness of a compressive device in controlling hemorrhage following radial artery catheterization.

Methods A prospective randomized trial was conducted on subjects receiving the standard taping method (group S) compared to a compressive device method (group C) after removal of the cannula in radial artery catheterization. Primary outcomes were the success rate of hemostasis and complication rate after cannula removal. Secondary outcomes were the cost of compression and the level of convenience.

Results A total of 250 subjects were enrolled in this study. Hemostasis after removal was successful in 122 of 125 (97.6\%) subjects in group $S$ and 116 of $125(92.8 \%)$ subjects in group C $(P=0.18)$. Complication rates in group $S$ and group $C$ were $55.2 \%(69 / 125)$ and $48 \%(60 / 125)$, respectively $(P=0.35)$. The cost of compression for group $C$ (approximately 6,740 Korean won) was approximately two times cheaper than for group $S$ (approximately 14,140 Korean won). The level of convenience was significantly higher in group $C$ than in group $S(7.4 \pm 2.1$ vs. $3.7 \pm 1.9$, $\mathrm{p}<0.001)$.

Conclusion These findings suggest that hemostasis using a compressive device may be a suitable alternative method to the standard taping method in controlling hemorrhage following radial artery cannulation.

Keywords Radial artery; Invasive techniques; Hemostasis; Equipment and supplies

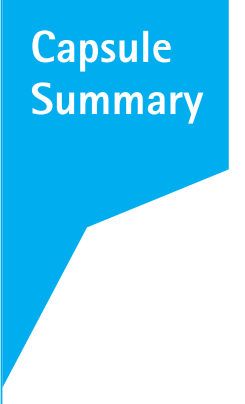

What is already known

Taping and physical compression is a traditional hemostatic method to be performed by the medical staff for 10 to 15 minutes after removal of radial artery cannula. Recently, hemostatic compressive devices have become widely used, replacing the traditional standard taping method to enable quick recovery and safe discharge following radial artery compression.

What is new in the current study

This randomized controlled trial study shows that the convenience of the compressive device for the providers as well as the cost-effectiveness were better than that with the standard method.
elSSN: 2383-4625

Received: 5 July 2014

Revised: 11 January 2015

Accepted: 17 February 2015

Correspondence to: Giwoon Kim Department of Emergency Medicine, Ajou University School of Medicine, 206 World cup-ro, Yeongtong-gu,

Suwon 443-721, Korea

E-mail: flyingguy@ajou.ac.kr

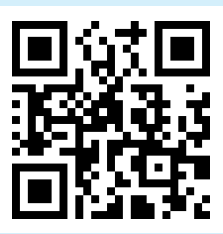

How to cite this article:

Yun K, Jeon W, Kang B, Kim G. Effectiveness of a compressive device in controlling hemorrhage following radial artery catheterization. Clin Exp Emerg Med 2015;2(2):104-109.

This is an Open Access article distributed under the terms of the Creative Commons Attribution Non-Commercial License (http:// creativecommons.org/licenses/by-nc/3.0/). 


\section{INTRODUCTION}

Arterial catheterization is frequently used in the management of critically ill patients. This procedure is usually easily accomplished at the bedside, using percutaneous methods such as the Seldinger technique to cannulate the radial, brachial, axillary, femoral, or dorsalis pedis artery. ${ }^{1}$ The consistent anatomic accessibility of the radial artery, ease of performing cannulation, and low rates of complications make it the preferred site for arterial cannulation. ${ }^{2}$ Complications after radial cannulation include bleeding (0.5\%), hematoma (14.4\%), pseudoaneurysm (0.09\%), and transient vascular occlusion (19.7\%). ${ }^{2}$ After cannula removal during radial artery cannulation (RAC), proper compression of the cannulation site is essential to prevent subsequent catheterization-related complications.

Taping and physical compression is a traditional hemostatic method that is most commonly used to achieve successful hemostasis and reduce the incidence of complications after removal of the cannula. However, this method involves labor costs. After removing the cannula in RAC, it is usual for compression to be performed by the medical staff for 10 to 15 minutes. Recently, hemostatic compressive devices have become widely used, replacing the traditional standard taping method to enable quick recovery and safe discharge following radial artery compression..$^{3-6}$ Considering patient crowding and the relative shortage of human resources in the emergency department, compressive devices could be useful for preventing hemorrhage following RAC.

Therefore, we conducted a prospective randomized trial to compare the effectiveness of compressive devices for the hemostasis rate and incidence of complications following cannula removal in RAC. The hypothesis underlying this study was that using a compressive device method would have a similar hemostasis and complication rate after cannula removal in RAC and be more cost effective and convenient than the standard taping method.

\section{METHODS}

A total of 250 consecutive patients aged 20 years or more who underwent radial cannulation in the operating room and then had the cannula removed in the recovery room from September 1 to December 31, 2010 were enrolled in this study. Subjects who had bleeding disorders or who were taking medications that could increase their hemorrhagic tendency were included, but those who had infection at the cannulation site were excluded, as were subjects who were discharged from the hospital prior to 1 week of follow-up. This study was approved by the institution review board (AJIRB-DEV-DE1-10-199). Written informed consents were obtained from all subjects or their legal representative.

A workshop session outlining the study was conducted for the medical professionals who performed the removal of the radial artery cannula from the subjects. The explanation included how to apply tape to fix the radial cannulation site after the standard taping method for 10 minutes and how to use a hemostatic compressive device. A practical exercise was performed on a mock patient. For 1 week prior to the start of our study, both methods were practiced on real patients. Using a random-numbers table, the patients were randomized to group $S(n=125)$, in which the standard taping method was applied, and group $C(n=125)$, in which the hemostatic compressive device was applied (Fig. 1).

Primary outcomes were the success rate of hemostasis and the complication rate after cannula removal; secondary outcomes were the cost of compression and the level of practitioner convenience. In group $\mathrm{S}$, the site of RAC was manually compressed for 10 minutes using sterilized gauze or an alcohol cotton ball, and then gauze was placed and tape was applied for fixation (Fig. 2A). In group $C$, the hemostatic compressive device was placed on the RAC site, the compression pad was held in place, and the cannula was then removed. Compression was applied using an adhesive strap (Fig. 2B). In both groups, compression was loosened after 15 minutes of fixation for 1 minute to observe the cannulation site for any bleeding or swelling. If no bleeding or swelling was observed, then the compression was halted. If bleeding or swelling was observed, then the standard taping method was applied for another 5 to 10 minutes until hemostasis was achieved and recorded as failure of hemostasis. After removing the radial cannula, the levels of satisfaction of both methods were graded from 0 (inconvenient) to 10 (convenient). One day after cannula removal, we checked whether any complications related to the cannulation site (e.g., bleeding, ecchymosis, hematoma, pseudoaneurysm formation, or arterial occlusion) occurred. The follow-up examination was performed 1 week after cannula removal. Bleeding was

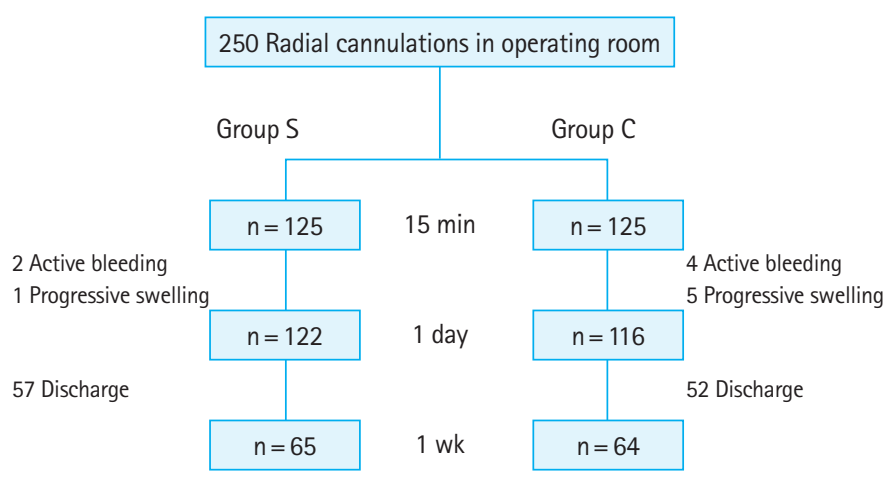

Fig. 1. Subject flow. Group S, standard tape method group; group C, compressive device method group. 


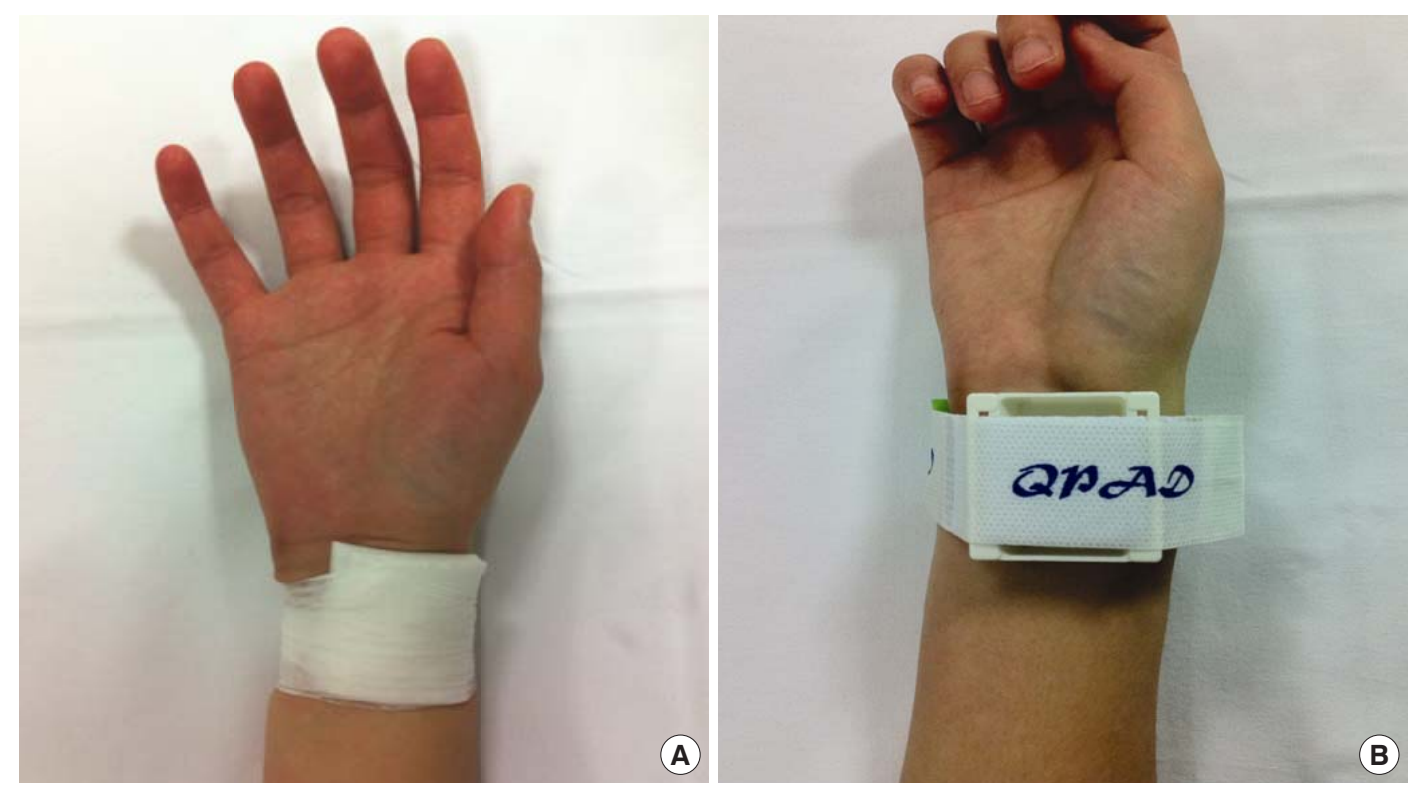

Fig. 2. Hemostasis methods after the removal of radial artery cannulation. The site of radial artery cannulation was manually compressed for 10 minutes using sterilized gauze or an alcohol cotton ball, and then gauze was placed and tape was applied for fixation (group S, standard method; A). The compressive device was placed on the radial artery cannulation site, the compression pad was held in place, and the cannula was then removed. Compres-

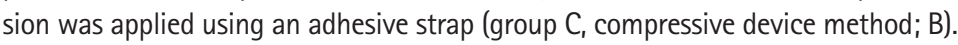

defined as any active bleeding at the radial cannulation site. Ecchymosis was defined as a change in skin color due to the presence of a blood deposit in the subcutaneous tissue without swelling at the cannulation site. Hematoma was defined as the simultaneous presence of ecchymosis and swelling at the cannulation site. A pseudoaneurysm was defined as observation of a thrill via visual inspection or of a bruit via a stethoscope. If the pulse was not palpable over the radial artery at the cannulation site, then the ipsilateral ulnar artery was compressed for 5 minutes using the modified Allen test based on oxygen saturation. A pulse oximetry device (C30 plus; Charmcare, Seoul, Korea) was attached to the index finger of the ipsilateral arm, and if the oxygen saturation decreased by $5 \%$ or more from baseline, then arterial occlusion was considered to have occurred. ${ }^{7-9}$ Past medical histories of the subjects were investigated using their medical records. The results of blood tests (prothrombin time, partial thromboplastin, and platelet count) performed 12 hours before the removal of the cannula were also compared.

The cost in group $\mathrm{S}$ was calculated using the period of compression by medical staff ( 10 minutes) based on the salary per hour for family and general practitioners according to the 2013 US Bureau of Labor Statistics data (http://www.bls.gov); the cost in group $C$ was based on the price of the compressive device.

Statistical analyses were performed with SPSS ver. 15.0 (SPSS Inc., Chicago, IL, USA). The data were tested for normality and are expressed as mean \pm standard deviation. Comparisons of continu- ous data were made using t-tests. Categorical data were analyzed using chi-square tests. A 5\% significance level (two-tailed) was used for all analyses. A multiple logistic regression test was used for analyzing the effect of predictor variables on successful hemostasis. A sample size calculation (assuming that $a=0.05$ and $\beta=0.20$ with a $20 \%$ difference in response rates) indicated that the minimum required number of subjects for each group was 95.

\section{RESULTS}

\section{Subject characteristics}

The sex, age, medical history, medication, prothrombin time, and partial thromboplastin time were similar between the two groups, whereas RAC durations and platelet counts were statistically different (Table 1). Multiple regression analysis, however, showed that the RAC duration and platelet counts did not affect the success of hemostasis at 15 minutes or any complications occurring at 1 day or 1 week after cannula removal.

\section{Main results}

Successful hemostasis at 15 minutes was observed in 95.2\% (238/ $250)$ of the subjects, active bleeding in $2.4 \%$ (6/250), and swelling in 2.4\% (6/250). One hundred twenty-two subjects in group S had achieved successful hemostasis at 15 minutes after cannula removal, 2 had active bleeding, and 1 had swelling, while 116 sub- 
Table 1. Subject characteristics

\begin{tabular}{llccc}
\hline Characteristics & & $\begin{array}{c}\text { Standard } \\
\text { method }\end{array}$ & $\begin{array}{c}\text { Compressive } \\
\text { device method }\end{array}$ & P-value \\
\hline Male & $69(55.2)$ & $61(48.8)$ & 0.31 \\
Age (yr) & $57 \pm 17.7$ & $59 \pm 15.1$ & 0.29 \\
Past medical & None & 50 & 43 & 0.52 \\
history & Liver disease & 0 & 1 & \\
& Hypertension & 50 & 54 & \\
& Diabetes mellitus & 34 & 32 & \\
Hematologic & Hemorrhagic disease & 6 & 10 & \\
study & PT (sec) & $11.1 \pm 1.07$ & $11.2 \pm 0.86$ & 0.77 \\
& PT (INR) & $0.98 \pm 0.09$ & $0.98 \pm 0.07$ & 0.81 \\
\multirow{5}{*}{ Duration of RAC } & PTT (sec) & $30.4 \pm 20.8$ & $27.4 \pm 3.1$ & 0.12 \\
\hline
\end{tabular}

Values are presented as number (\%) or mean \pm standard deviation.

PT, prothrombin time; INR, international normalized ratio; PTT, partial thromboplastin time; RAC, radial artery cannulation.

${ }^{*} \mathrm{P}<0.05$.

Table 2. Comparison of the standard and compressive device methods following removal of the radial artery cannula

\begin{tabular}{clrrc}
\hline $\begin{array}{c}\text { Time after } \\
\text { cannula } \\
\text { removal }\end{array}$ & Complications & Standard & Compressive & P-value \\
\hline 15 minutes & None & $122(0.98)$ & $116(0.93)$ & - \\
Minutes & Active bleeding & $2(0.02)$ & $4(0.03)$ & - \\
& Progressive swelling & $1(0.01)$ & $5(0.04)$ & 0.18 \\
1 Day & None & $53(0.42)$ & $56(0.45)$ & - \\
& Ecchymosis & $49(0.39)$ & $46(0.37)$ & - \\
& Hematoma & $17(0.14)$ & $14(0.11)$ & - \\
& Occlusion & $3(0.02)$ & $0(0.00)$ & 0.35 \\
1 Week & None & $36(0.29)$ & $46(0.37)$ & - \\
& Ecchymosis & $29(0.23)$ & $17(0.14)$ & - \\
& Hematoma & $0(0.00)$ & $1(0.01)$ & 0.07 \\
\hline
\end{tabular}

jects in group $\mathrm{C}$ had achieved successful hemostasis at $15 \mathrm{~min}-$ utes after cannula removal, 4 had active bleeding, and 5 had swelling $(P=0.18)$. Regarding the variables for the first day post-removal, no complications were observed in 109 subjects (45.7\%), ecchymosis was observed in 95 (39.9\%), hematoma in 31 (13.0\%), and vascular occlusion in $3(1.2 \%)$. Regarding the variables for 1 week post-removal, 57 subjects in group $S$ and 52 subjects in group $C$ were excluded from the study. Of the remaining subjects $(n=129)$, 82 (63.5\%) were without complications, while 46 (35.6\%) had ecchymosis, and $1(0.7 \%)$ had hematoma. The 3 subjects who had developed vascular occlusion at the first day post-removal were found to have normal modified Allen test results. No pseudo-aneurysms were observed.

Regarding the variables for the first day post-removal, 53 subjects in group S were still without any complications, but 49 had ecchymosis, 17 had hematoma, and 3 had vascular occlusion. Fif-

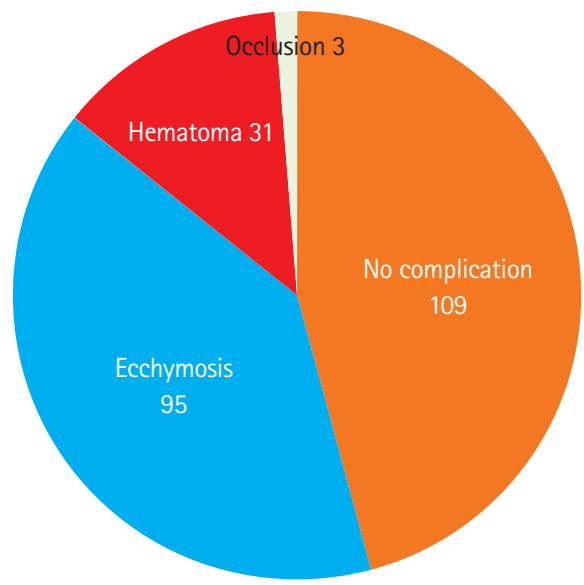

Fig. 3. Complications at 1 day after the removal of radial arterial cannulation.

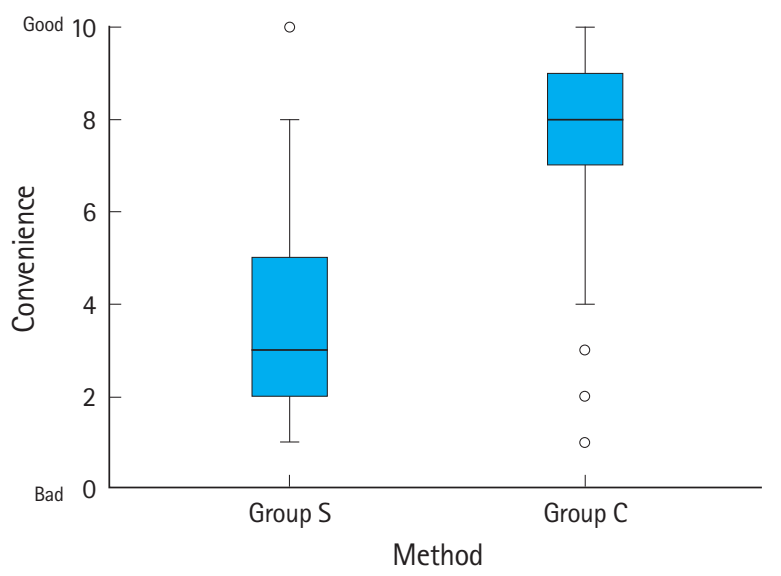

Fig. 4. Comparison of convenience between the $\mathrm{S}$ group and the $\mathrm{C}$ group.

ty-six subjects in group C were still without complications, but 46 had ecchymosis, 14 had hematoma, and none had vascular occlusion ( $P=0.345$ ) (Table 2, Fig. 3). Regarding the variables for 1 week post-removal, 36 subjects in group $S$ were found to have no complications, but 29 had ecchymosis. In group C, 46 subjects did not have complications, 17 had ecchymosis, and 1 had hematoma ( $P=0.07$ ) (Table 2). The 3 subjects who had developed vascular occlusion at the first day post-removal were found to have a normal modified Allen test 1 week later, suggesting that the occlusion was transient.

The costs for group $S$ and group $C$ were about 14,140 and 6,740 Korean won, respectively, thus the cost of group $S$ was about twice as expensive as group $C$. The levels of convenience in group $S$ and group $C$ were $3.7 \pm 1.9$ and $7.4 \pm 2.1$, respectively $(P<0.001)$ (Fig. 4). 


\section{DISCUSSION}

We found that hemostasis using the compressive device may be a suitable alternative method comparable to the standard taping method in controlling hemorrhage following RAC. The complications associated with RAC included bleeding (0.5\%), hematoma (14.4\%), pseudoaneurysm (0.1\%), transient vascular occlusion $(19.7 \%)$, and permanent ischemic damage (0.1\%). ${ }^{2}$ Despite these events, RAC was a safe procedure, since pseudoaneurysm and ischemic damage requiring surgery and treatment almost never occurs. ${ }^{2,10,11}$ In the present study, transient vascular occlusion occurred in 3 subjects, but no ischemic symptoms were observed; moreover, the modified Allen test performed at 1 week post-removal showed that the subjects had no abnormalities. For cases of permanent ischemic damage in the distal part of the hand due to vascular injury or thrombotic events occurring after RAC, treatment using vasodilators are reported to be effective. ${ }^{12}$

The compression device has several advantages over traditional physical compression. First, it can be helpful in preventing potential vascular occlusions, because the newly developed compressive devices are designed to maintain the radial artery blood flow (according to the product manual) when compression is required for hemostasis at the wrist. Occlusive radial artery compression has also been reported to be a risk factor for subsequent radial artery occlusion. Vascular occlusions are reported to occur in $25 \%$ to $30 \%$ of patients after RAC when a $20 \mathrm{G}$ Teflon cannula (commonly used in the operating room or intensive care unit) is used. . $^{1314}$ In contrast, when $6 \mathrm{Fr}$ cannulas are placed in the radial artery for coronary angiography, transient radial artery occlusions are reported to occur in only $5.3 \%$ of patients. ${ }^{15,16}$ Since it is difficult to apply reliable compression pressure when the tape is used as a fixation mechanism for hemostasis after cannula removal, it is likely that compression can block the radial artery blood flow; we infer that transient vascular occlusion may have occurred in the 3 subjects receiving standard treatment because of this blockage. Second, it is more convenient for medical staff than is traditional physical compression, because the standard physical method requires physical effort from the medical staff. Third, it might prevent the ipsilateral hand from swelling during the period of compression. The use of tape for continuous compression against the cannulation site can result in swelling of the hand by interrupting venous blood flow. However, the compressive device is designed to maintain appropriate compression for an extended period against the RAC site while maintaining venous blood flow through the multiple grooves of the pad that come in contact with the dorsum of the wrist. In addition, because the compression device has a blunt and soft surface, it is likely to be more comfort- able and better tolerated. Fourth, the economic feasibility will be remarkable in the scope of labor management. When the compressive device is applied in post-removal compression, the total cost can decrease to half the cost of the traditional compression method, which is labor-intensive. Opportunity costs for the labor of medical staff, including doctors, nurses, and clinical assistants would be available to perform other functions. In today's health care environment, labor expense is high at up to 50\% of a healthcare system's annual budget. Therefore, effective labor management is needed for hospitals, regardless of their facility size or internal technical expertise.

The limitations of this study are as follows. First, the sample size was small, as indicated by the 20\% difference in the success rate between the two groups, which might bias the results. Despite this problem, this was conducted as a prospective randomized controlled study. Therefore, we believe this issue did not significantly affect the findings. Second, the pain and convenience that the patients experienced were not considered a variable as the mental status of the subjects in the recovery room was not clear. Further study is needed to clarify this issue. Third, patients with a long RAC time were not included because the study was performed on subjects who underwent short-duration operations (mean, 195 \pm 77.7 minutes). Fourth, the degrees of vascular injury that may have occurred during the procedures were not identified since the numbers of RAC attempts and the successes or failures of the RAC were not investigated.

In conclusion, the success rate of hemostasis at 15 minutes for the compressive device was not different from the standard taping method. The incidence of complications was also not different from the standard method. In addition, the convenience of the compressive device for the providers as well as the cost-effectiveness were better than that with the standard method. These findings suggest that the compressive device may be an effective alternative method to the standard taping method in controlling hemorrhage following RAC.

\section{CONFLICT OF INTEREST}

No potential conflict of interest relevant to this article was reported.

\section{ACKNOWLEDGMENTS}

The compression device was used in this study was provided by IMT Medical Co. 


\section{REFERENCES}

1. Clark VL, Kruse JA. Arterial catheterization. Crit Care Clin 1992; 8:687-97.

2. Scheer B, Perel A, Pfeiffer UJ. Clinical review: complications and risk factors of peripheral arterial catheters used for haemodynamic monitoring in anaesthesia and intensive care medicine. Crit Care 2002;6:199-204.

3. Agostoni P, Biondi-Zoccai GG, de Benedictis ML, et al. Radial versus femoral approach for percutaneous coronary diagnostic and interventional procedures: systematic overview and meta-analysis of randomized trials. J Am Coll Cardiol 2004; 44:349-56.

4. Cubero JM, Lombardo J, Pedrosa C, et al. Radial compression guided by mean artery pressure versus standard compression with a pneumatic device (RACOMAP). Catheter Cardiovasc Interv 2009;73:467-72.

5. Rathore $\mathrm{S}$, Stables $\mathrm{RH}$, Pauriah $\mathrm{M}$, et al. A randomized comparison of TR band and radistop hemostatic compression devices after transradial coronary intervention. Catheter Cardiovasc Interv 2010;76:660-7.

6. Louvard $Y$, Benamer $H$, Garot $P$, et al. Comparison of transradial and transfemoral approaches for coronary angiography and angioplasty in octogenarians (the OCTOPLUS study). Am J Cardiol 2004;94:1177-80.

7. Brzezinski M, Luisetti T, London MJ. Radial artery cannulation: a comprehensive review of recent anatomic and physiologic investigations. Anesth Analg 2009;109:1763-81.

8. Fuhrman TM, Pippin WD, Talmage LA, Reilley TE. Evaluation of collateral circulation of the hand. J Clin Monit 1992;8:28-32.

9. Barbeau GR, Arsenault F, Dugas L, Simard S, Lariviere MM. Evaluation of the ulnopalmar arterial arches with pulse oximetry and plethysmography: comparison with the Allen's test in 1010 patients. Am Heart J 2004;147:489-93.

10. Hausmann D, Schulte am Esch J, Fischdick G. Radial artery cannulation: a prospective study on its complication rate by clinical and sonographic evaluation (author's transl). Anasth Intensivther Notfallmed 1981;16:269-73.

11. Slogoff $S$, Keats AS, Arlund C. On the safety of radial artery cannulation. Anesthesiology 1983;59:42-7.

12. Valentine RJ, Modrall JG, Clagett GP. Hand ischemia after radial artery cannulation. J Am Coll Surg 2005;201:18-22.

13. Bedford RF, Wollman H. Complications of percutaneous radial-artery cannulation: an objective prospective study in man. Anesthesiology 1973;38:228-36.

14. Davis FM, Stewart JM. Radial artery cannulation: a prospective study in patients undergoing cardiothoracic surgery. $\mathrm{Br} J$ Anaesth 1980;52:41-7.

15. Stella PR, Kiemeneij F, Laarman GJ, Odekerken D, Slagboom T, van der Wieken R. Incidence and outcome of radial artery occlusion following transradial artery coronary angioplasty. Cathet Cardiovasc Diagn 1997;40:156-8.

16. Pancholy S, Coppola J, Patel T, Roke-Thomas M. Prevention of radial artery occlusion-patent hemostasis evaluation trial (PROPHET study): a randomized comparison of traditional versus patency documented hemostasis after transradial catheterization. Catheter Cardiovasc Interv 2008;72:335-40. 